\title{
Upscaling of Permanent Magnet Dismantling and Recycling through VALOMAG Project ${ }^{\dagger}$
}

\author{
Fernando Coelho ${ }^{1, *} \mathbb{C}$, Shoshan Abrahami ${ }^{1}$, Yongxiang Yang ${ }^{1}$, Benjamin Sprecher ${ }^{2} \mathbb{D}, \mathrm{Zhijie} \mathrm{Li}^{3}$, \\ Nour-Eddine Menad ${ }^{4}$, Kathy Bru ${ }^{4}$, Thibaut Marcon ${ }^{5}$, Cyril Rado ${ }^{6}$, Boris Saje ${ }^{7}$, Marie-Lise Sablayrolles ${ }^{8}$ \\ and Virginie Decottignies ${ }^{8, *}$
}

1 Department of Materials Science and Engineering, T.U. Delft, 2628 CD Delft, The Netherlands; s.t.abrahami@tudelft.nl (S.A.); y.yang@tudelft.nl (Y.Y.)

2 Department of Sustainable Design Engineering, T.U. Delft, 2628 CD Delft, The Netherlands; sprecher@cml.leidenuniv.nl

3 Institute of Environmental Sciences (CML), Leiden University, 2333 CC Leiden, The Netherlands; z.li@cml.leidenuniv.nl

4 Bureau de recherches géologiques et minières (BRGM), 45060 Orléans, France; n.menad@brgm.fr (N.-E.M.); k.bru@brgm.fr (K.B.)

5 CRM Group, 4000 Liège, Belgium; thibaut.marcon@crmgroup.be

6 CEA (Commissariat à l'énergie atomique et aux énergies alternatives), 38000 Grenoble, France; cyril.rado@cea.fr

7 Kolektor, 5280 Idrija, Slovenia; boris.saje@kolektor.com

$8 \quad$ SUEZ, 92040 Courbevoie, France; marie-lise.sablayrolles@suez.com

* Correspondence: f.a.p.coelho-1@tudelft.nl (F.C.); virginie.decottignies@suez.com (V.D.)

+ Presented at International Conference on Raw Materials and Circular Economy, Athens, Greece, 5-9 September 2021.

Citation: Coelho, F.; Abrahami, S.; Yang, Y.; Sprecher, B.; Li, Z.; Menad, N.-E.; Bru, K.; Marcon, T.; Rado, C.; Saje, B.; et al. Upscaling of Permanent Magnet Dismantling and Recycling through VALOMAG Project. Mater. Proc. 2021, 5, 74. https://doi.org/ $10.3390 /$ materproc2021005074

Academic Editor: Konstantinos Simeonidis

Published: 10 December 202

Publisher's Note: MDPI stays neutral with regard to jurisdictional claims in published maps and institutional affiliations.

Copyright: (c) 2021 by the authors. Licensee MDPI, Basel, Switzerland. This article is an open access article distributed under the terms and conditions of the Creative Commons Attribution (CC BY) license (https:// creativecommons.org/licenses/by/ $4.0 /)$.
Abstract: Neodymium-Iron-Boron (NdFeB) based permanent magnets are indispensable in today's technology-driven society. Moreover, their use is likely to increase since they are key in clean energy applications such as wind turbines, hybrid/electric vehicles, and electric bikes. They contain critical raw materials as rare earth elements are used. Indeed, permanent magnets are considered strategic materials by the EU, and their recycling represents a potential secondary supply to decrease the import dependence. The VALOMAG project is developing a technical solution to recover rare earth (RE) based permanent magnets by dismantling end-of-life (EoL) products such as computer hard disc drives, electric motors, and generators from electric vehicles and wind turbines. It also assesses two short loop recycling technologies: Hydrogen Decrepitation (HD) or HydrogenationDisproportionation-Desorption-Recombination (HDDR) and strip-casting for high and medium quality magnet wastes; and hydrometallurgical processes for EoL low-quality magnets. Moreover, Life Cycle Assessment (LCA) and Process Integration with a Flowsheet simulation tool will integrate the whole recycling value chain (collection, dismantling, physical and chemical treatment options, and re-manufacturing) and assess the environmental impact and processes efficiency. A market study on the types and expected future quantities for the scrap magnets and the characterisation of the EoL magnets from hard disc drives (HDD) will be presented as preliminary results. Pre-treatment and sorting of 2.5 tons of $\mathrm{NdFeB}$ magnets scraps were carried out, and the two short loop recycling routes and the hydrometallurgical route are under investigation at the lab and pilot scale. The results will be used to develop a process integration and to assess the three routes through LCA.

Keywords: NdFeB; magnets; critical raw materials; dismantling; recycling

\section{Introduction}

Rare-Earth Elements (REEs) are becoming increasingly important in the transition to a green economy due to their essential role in permanent magnets, lamp phosphors, catalysts, and rechargeable batteries, among other applications. For example, Neodynium $(\mathrm{Nd})$ and 
Dysprosium (Dy) are used in more than $85 \%$ of the permanent magnets (PM) in the industry. In its landmark report Critical Raw Materials for the European Union (2020) [1], the European Commission considers REEs as the most critical raw materials group, with the highest supply risk. The five most critical REEs are neodymium $(\mathrm{Nd})$, europium $(\mathrm{Eu})$, terbium (Tb), dysprosium (Dy), and yttrium (Y) [2]. Moreover, it is anticipated that over the next 25 years, the demand for $\mathrm{Nd}$ and Dy will rise by $700 \%$ and $2600 \%$, respectively, especially due to the increasing popularity of hybrid and electric vehicles $(\mathrm{H}) \mathrm{EV}$ and wind turbines [3].

REEs production is centralised in China (accounting for the global production of over $90 \%$ ), and exportation quotas have been imposed by the Chinese government, arousing concerns on the REEs supply risk at the European level. Furthermore, while mining companies are actively seeking new exploitable REEs deposits and old mines are being reopened, the construction of new mines is often associated with high capital costs and timelines of more than 10-15 years before they can start operating [4]. Thus, the recycling of REEs could be far more economical and more readily achievable than the exploitation of new mineral deposits.

Despite vast, mostly lab-scale research efforts on REEs recycling, up to 2018, less than $1 \%$ of the REEs were recycled, mainly due to inefficient collection, technological problems, and a lack of industries supporting the recycling process. Therefore, a drastic improvement in the recycling of REEs is an absolute necessity that can only be achieved by developing efficient, fully integrated recycling routes, which can take advantage of the rich REE recycling literature [5]. The most promising candidate for the so-called 'urban mining' is the end-of-life products containing $\mathrm{NdFeB}$ magnets.

From the recycling process technology, three available options have been applied to the recycling of $\mathrm{NdFeB}$ magnets: (1) direct reuse, (2) recycling of the permanent magnet alloy, and (3) elemental recycling, which means the extraction of REEs or oxides [6]. From an environmental and economic point of view, the most favourable is direct reuse, followed by alloy and elemental recycling. However, the magnets' characteristics, such as shape, dimensions, chemical compositions, magnetic properties, and required specifications, render the first option (reuse) often not feasible. On the other hand, keeping and recycling the magnetic alloy as a whole appears to be very attractive in terms of energy, environmental, and raw materials costs. At the same time, the elemental recycling from the more complex hydrometallurgy process can be used for a low-grade feed $[7,8]$.

The VALOMAG project proposes to supply a technical solution for permanent magnet disassembly of EoL products, such as HDDs, electric vehicles, and wind turbines, and to assess two short loop recycling technologies (HD/HDDR and strip casting) for high and medium quality magnets with a third alternative route using hydrometallurgical processes for low-quality magnets. The project combines different key players who bring together their expertise to develop a new value proposition that answers the need of the permanent magnets market, as shown in Figure 1.

The work presented in this paper is focused on the first phase of the project, which involves: (1) the sourcing of EoL HDD; (2) the thermal treatment; and (3) the fragmentation and classification of the different material fractions. 


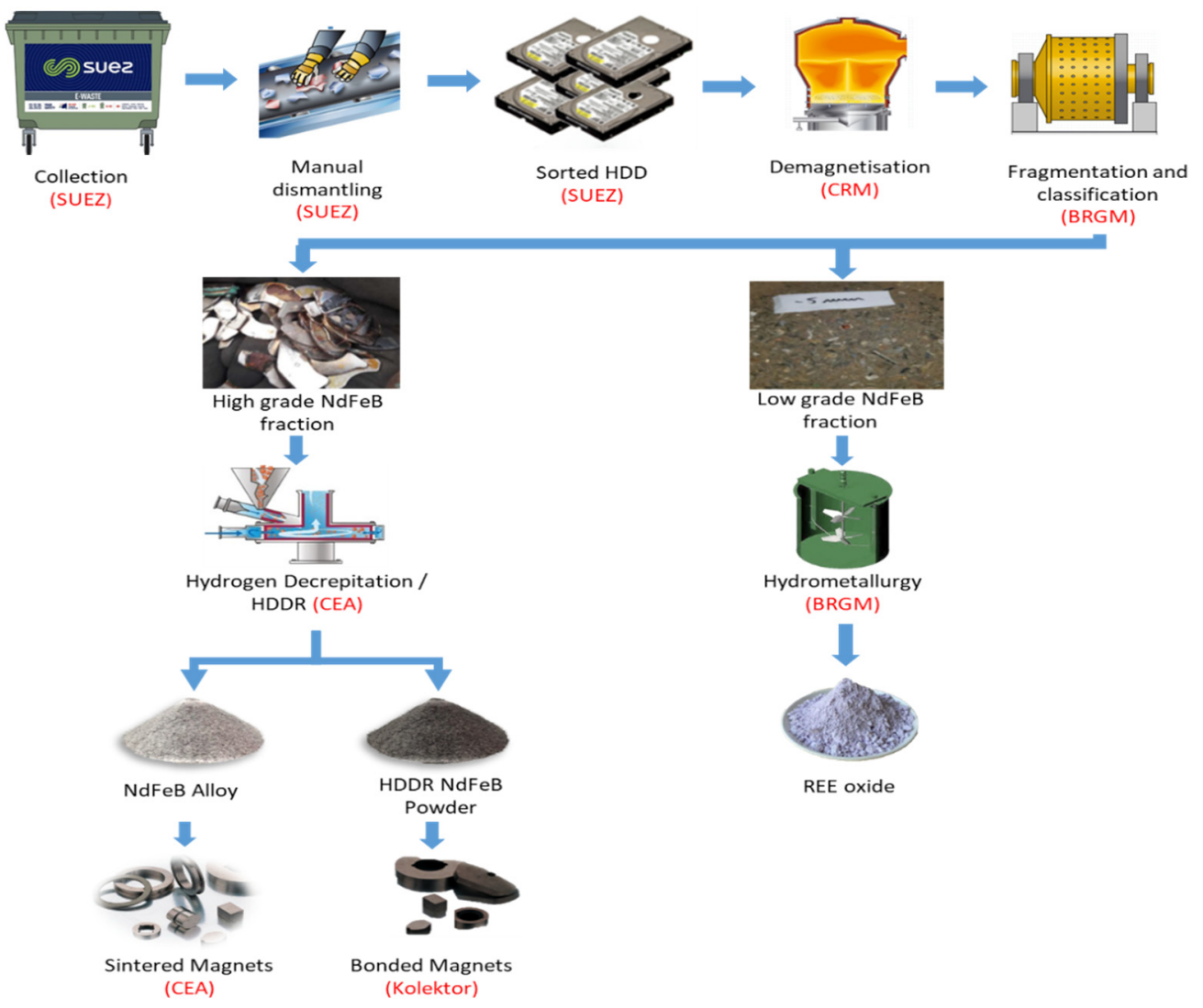

Figure 1. Process flow chart planed in VALOMAG initiative, with the involved partners in red.

\section{Materials and Methods}

\subsection{Sourcing of EoL HDDs}

The waste electrical and electronic equipment (WEEE) was sorted by the SUEZ WEEE dismantling plant in France via manual disassembly of desktop personal computers (PCs), allowing the extraction of the HDDs (see Figure 2). The input fraction corresponds to old appliances collected in the B2B WEEE scheme produced by businesses $(90 \%$ is currently collected). Therefore, this fraction is mainly composed of desktop computers. Two examples of 3.5-inch HDDs have been characterised by manual disassembly; they correspond to Western Digital brands.

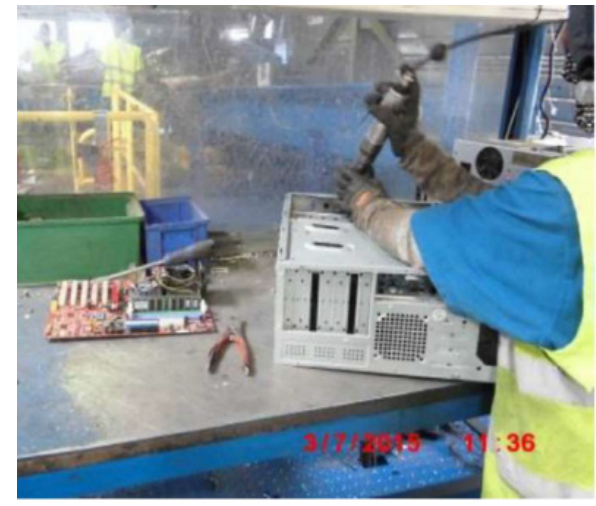

(a)

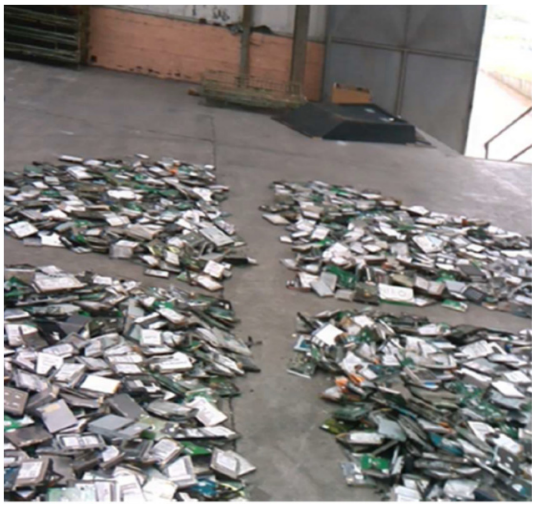

(b)

Figure 2. (a) Manual disassembly of the personal desk computers. (b) HDDs sorted for sampling. 


\subsection{Demagnetisation}

The HDDs collected were sent to CRM Group (Belgium) for thermal demagnetisation of the contained magnets. Two different tests were performed, considering two criteria, firstly the prevention of clogging and magnets adhesion issues in the subsequent equipment used for the dismantling process; secondly, the easier separation of the different components.

The objective of the 1st test trials was to observe the effect of heating on magnets and organic parts but, as the crucible size was limited, it was decided to manually disassemble some HDDs and put in the furnace only the useful parts (nearly all components except the casing and the screws). A slow heating process in an air atmosphere was chosen to allow temperature homogenisation in the sample without heating at too high a temperature and to avoid organics degradation. This standard heating process lasts $90 \mathrm{~min}$ at $300-350{ }^{\circ} \mathrm{C}$.

In the second test, the samples were exposed to a "thermal flashing" in an inert atmosphere, which corresponds to the opposite of a quench (cooling). Concretely, the sample still at ambient temperature is directly loaded in the furnace at high temperature, far above Curie temperature. Then, after a brief time (a few minutes) at a high temperature, the sample is quenched to stop the thermal diffusion within the elements and reduce organics degradation. The conditions were varied from $450{ }^{\circ} \mathrm{C}$ to $700{ }^{\circ} \mathrm{C}$ and for 6 to $15 \mathrm{~min}$.

\subsection{Fragmentation and Classification}

The fragmentation of HDDs (demagnetised) was carried out by BRGM (France), using an autogenous mill. The procedure was based on previous work developed by the same research group in the Extrade project [9]. The fragmented HDDs were classified into 1-5 mm, 5-20 mm, and 20-40 mm fractions. The $20-40 \mathrm{~mm}$ fraction (magnet rich) was separated by low intensity dry magnetic separation to recover all magnet pieces. The dry, low-intensity, magnetic separation was performed on size fractions of 5-20 mm and below $5 \mathrm{~mm}$ obtained from the ordinary fragmentation to concentrate the magnets. Three fragmentation tests were performed on hard disc drives using an autogenous mill. The first test deals with ordinary fragmentation (ORFg) for $45 \mathrm{~min}$ and the second consists of a sequential fragmentation (SQFg) of 3 steps of $15 \mathrm{~min}$ to avoid the overproduction of fine particles. The third trial involved sequential fragmentation into six fragmentation steps of $15 \mathrm{~min}$ each. As a result, fragmented HDDs have been classified at $40 \mathrm{~mm}$. The coarser size fraction was returned to the autogenous mill for the second milling and for a total processing time of $90 \mathrm{~min}$.

Figure 3 gives the global flow sheet of HDD magnets treatment, including sorting, demagnetisation, fragmentation, classification, and magnetic separation. 


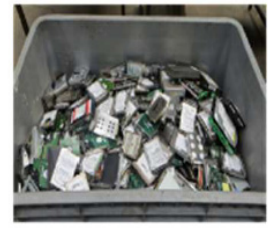

Hard Disc Drives

Collected by Suez
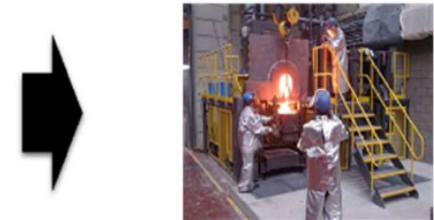

Demagnetisation CRM (induction furnace)
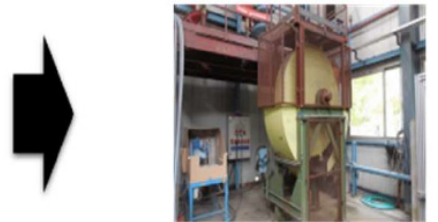

Fragmentation (autogenous mill)

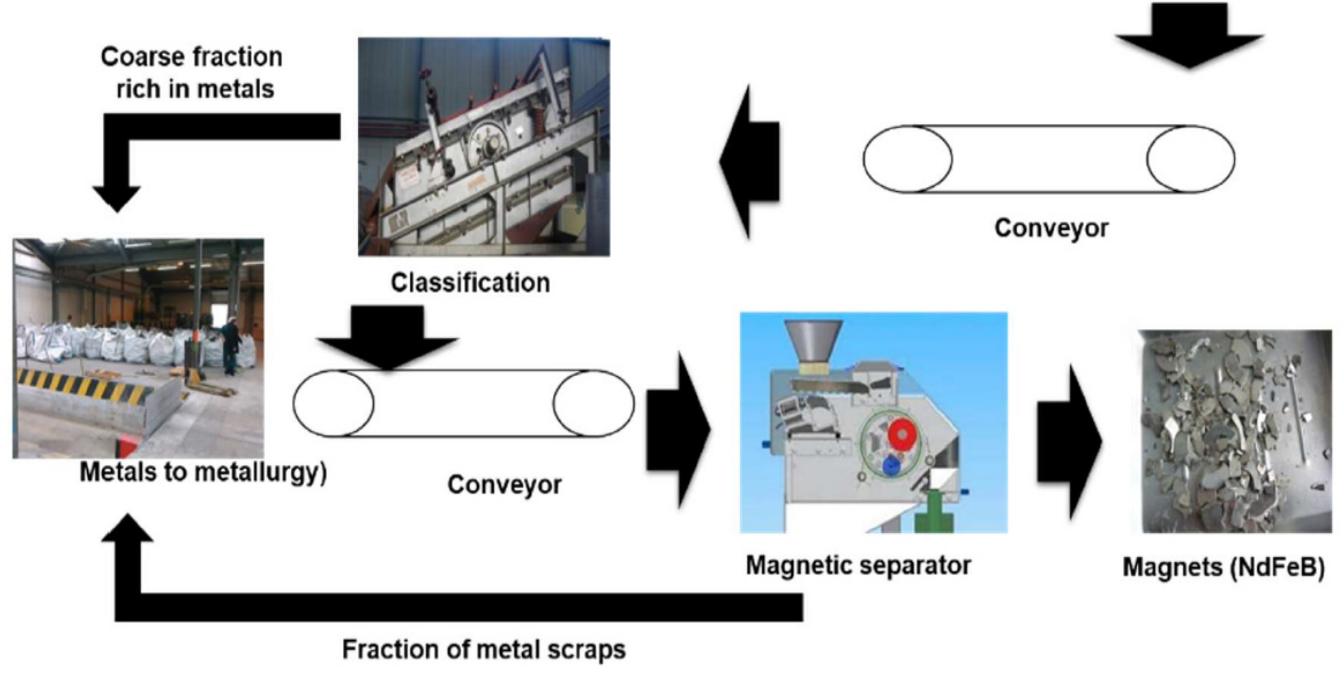

Figure 3. Global flowsheet of HDD magnets treatment.

\section{Results}

\subsection{Sourcing HDDs}

After the manual separation of all the extracted fractions, HDDs from the B2B collection, two different models of the 3.5-inch were dismantled. The HDDs and their components are shown in Figure 4. The WD800 3.5-inch HDD (Production date 2003), with an aluminium top cover, weighed $576.5 \mathrm{~g}$ in total with $11 \mathrm{~g}$ of sintered magnets from the voice coil motor. The WD800JD (Production date 2008) weighed $459.3 \mathrm{~g}$, with $6 \mathrm{~g}$ of sintered magnets from the voice coil motor and had a steel top cover.

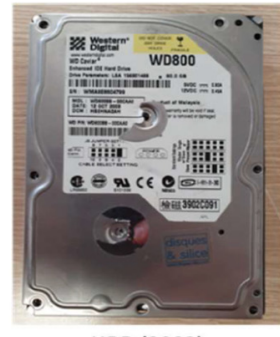

HDD (2003)

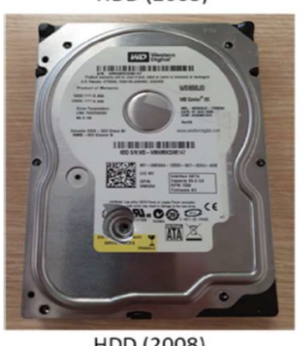

HDD (2008)
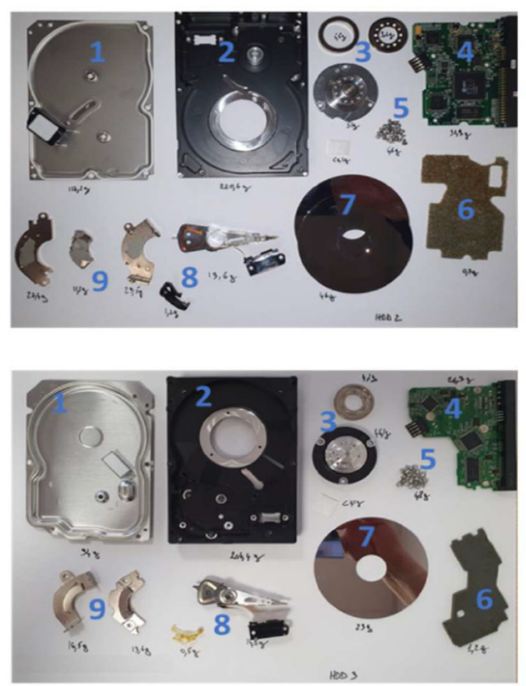

Elements of HDD:

1. Top cover

Mounting chassis

Spindle motor (1 bonded magnet)

PCB (Printed Circuit Board)

Screws

PU foam

Platter

Read/write head and head arm

2 sintered magnets assembly

from the voice coil motor

Figure 4. The 3.5" HDDs and disassembled components. 


\subsection{Demagnetization}

The results of the tests are shown in Table 1, with a comparison of the standard heating process (left grey frame of the table) and the "flash heating" conditions (the right frame of the table).

Table 1. Demagnetisation tests.

\begin{tabular}{|c|c|c|c|c|c|c|c|c|c|c|c|c|}
\hline Temperature $\left[{ }^{\circ} \mathrm{C}\right]$ & 240 & 250 & 260 & 280 & 305 & 350 & 450 & 550 & 550 & 600 & 650 & 700 \\
\hline Time [min] & 90 & 120 & 90 & 90 & 90 & 90 & 15 & 15 & 8 & 6 & 7 & 8 \\
\hline Demagnetisation & $\mathrm{X}$ & $\mathrm{X}$ & $X$ & $X$ & $\sqrt{ }$ & $\sqrt{ }$ & $X$ & $\sqrt{ }$ & $X$ & $X$ & $X$ & $\sqrt{ }$ \\
\hline Plastic Degradation & $\sqrt{ }$ & $\sqrt{ }$ & - & - & - & - & $\sqrt{ }$ & - & $\sqrt{ }$ & $\sqrt{ }$ & - & 一 \\
\hline
\end{tabular}

In the table, the grey box (left) represents the first batch test and the right-side thermal flash batch. " $\sqrt{ }$ " indicates successful results in terms of quality recovery for the different materials; " $\mathrm{X}$ " indicates non-demagnetised magnets, and the number of "-" symbols indicate the level of plastic degradation (-, low degradation, - , high degradation).

The 1st trials (in grey) did not reach the expectations. Indeed, no settings granted a full demagnetisation and good preservation of the organics simultaneously. However, the results were useful to find the degradation of the organic occurring at $170{ }^{\circ} \mathrm{C}$, which make it impossible to find a thermal window between the Curie temperature and organic degradation temperature, as shown in Figure 5.

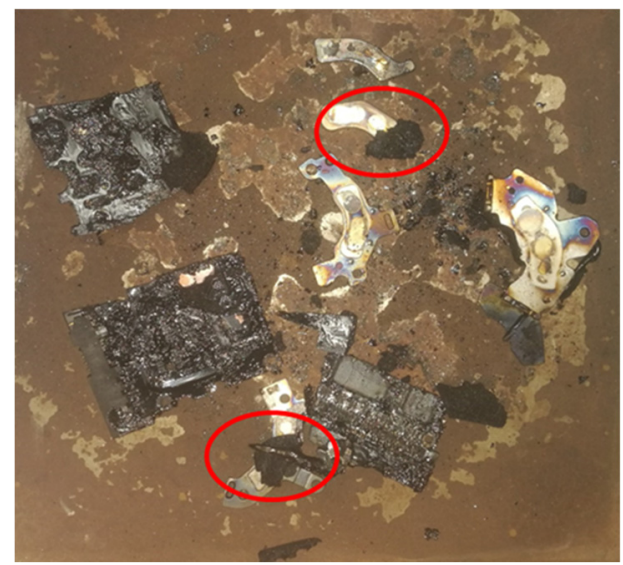

Figure 5. Example of components observed after thermal treatment at $305^{\circ} \mathrm{C}$ during $90 \mathrm{~min}$. With the red circles representing the burnt organic parts over metallic elements.

The 2nd series of tests gave better visual results on the different components of the HDD. Even if further optimisation could be required, a significant improvement has been observed in the quality of materials recovered after thermal treatment with a complete demagnetisation leading to an easier dismantling process.

\subsection{Fragmentation and Classification}

The materials that were manually sorted by particle size classes $(80,40,20,14$, and $5 \mathrm{~mm}$ ) obtained from the classification of fragmented HDDs for the three pilot tests were weighed. The percentage distributions were calculated, and the results are illustrated in Figure 6. This figure shows that most iron scraps were present in both the 80- and 40-mm size fractions for the three pilot tests. Pieces of aluminium and PCB were found in the coarse fraction. This confirms that the autogenous mill did not shred these parts, which is good for a further recovery process. Almost all the magnets were present in the fine fractions because, after heating, the magnets became brittle and easy to break. Copper wires were found in the 20- and 14-mm size fractions. Some parts that were not released were present in the coarse fraction; they need to be shredded for further processing. 

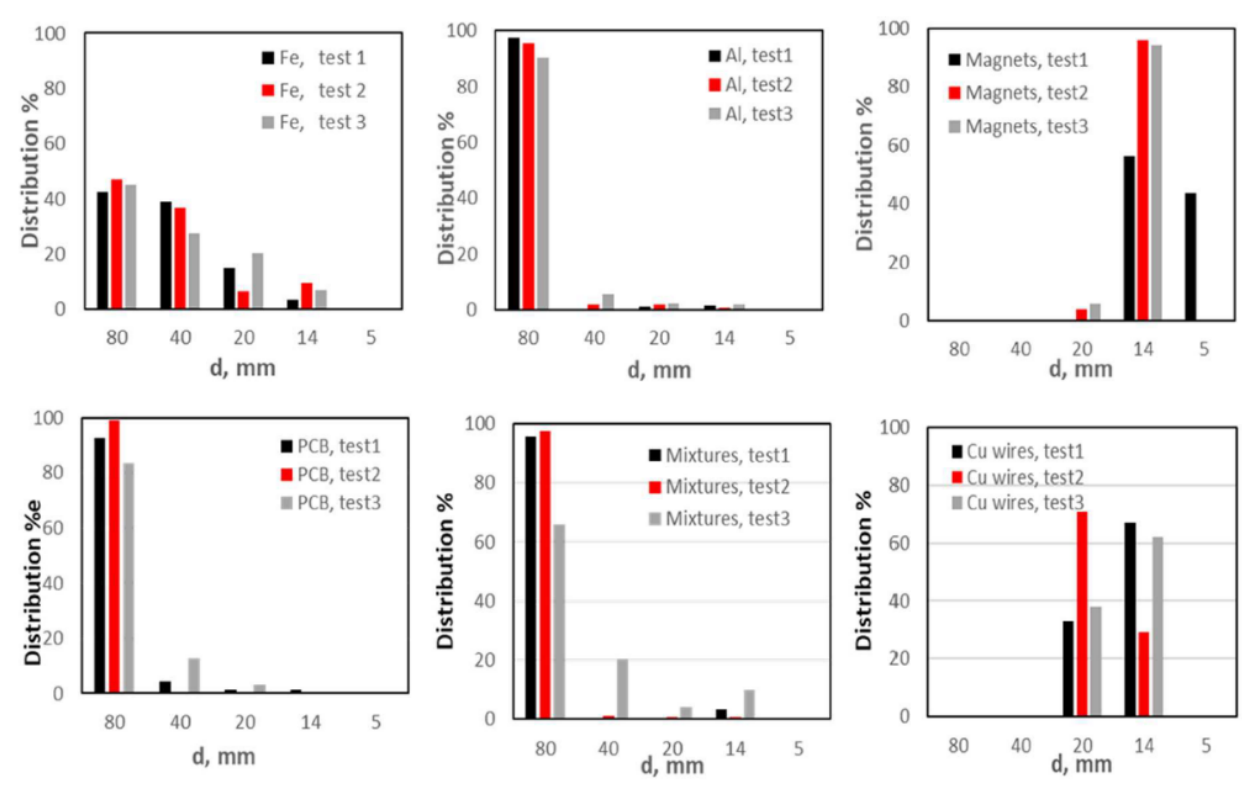

Figure 6. Percentage distribution of hard drive materials manually sorted into 80-, 40-, 20-, 14-, and 5-mm size fractions obtained from the three pilot tests.

\section{Discussion/Conclusions}

The Hard Disk Drives (HDDs) are a major component of desktop and laptop computers. HDDs are used for secondary storage of data, and NdFeB magnets are used to spin the drive where the data are stored. The use of HDDs for storage in data centre (cloud) applications will increase by 3.5 HDDs and provide the industry with a growth opportunity in 2021 to support remote working. Nevertheless, in the medium and long term, the flows of WEEE containing $\mathrm{NdFeB}$ are expected to increase. As a result, their collection rate and new recycling processes for REEs are likely to emerge, covering an important additional part of REE demand. The composition of the HDDs has been described by other authors [7,9-11] and are in line with the results shown in this paper.

The thermal treatment is key in the pre-processing steps, avoiding accretion and clogging in the autogenous grinder during the fragmentation step. Thermal treatment can also impact the other materials present in EoL products, leading to less quantity of materials recovered if damaged during the thermal process. An experimental plan has been implemented to test different heating techniques and assess different parameters, leading to an innovative solution dedicated to HDDs. The process allowed the HDD to be treated while respecting the quality expectations and adding some positive side effects (patent under development). The setting campaigns highlighted the physical limits of a classical heating process to reach the two mandatory criteria for the thermal treatment of HDD: full demagnetisation of the inner magnets and limited degradation of organic materials, including the PCBs. If organics become volatile, a post-combustion treatment will be required, or if they melt, they will cover other components and reduce the quality of the separation. The degradation of PCBs will also lead to reducing the recovery of other materials and metals from the HDDs. It was also observed that the Curie temperature (between $300^{\circ} \mathrm{C}$ and $370^{\circ} \mathrm{C}$ ) is highly affected by the magnets' compositionwith the presence and concentration of Praseodymium (Pr) significantly impacting the Curie temperature.

Regarding the HDDs fragmentation, the SQFg performed better than ORFg, and no difference is observed in the particle size distribution of the fractions obtained from the classification of fragmented hard drives. The PCBs (99\%) were mostly present in the coarse fraction $(40-80 \mathrm{~mm})$, and about $97.5 \%$ of magnets were distributed in the size fraction 5-14 mm; the magnet content of this size fraction being $57 \%$. The autogenous mill did not shred the large parts, which is good for a further recovery process. Almost all the magnets were present in fine fractions because, after their heating, the magnets became brittle and 
easy to shred. Some parts not released were present in the coarse fraction; they need to be shredded for further processing.

Valomag project is developing a technical solution to recover permanent magnets by dismantling end-of-life (EoL) products. For the next phase of the project, the two short loop recycling technologies will be developed: Hydrogen Decrepitation (HD) or Hydrogenation-Disproportionation-Desorption-Recombination (HDDR) and strip-casting high and medium quality magnet wastes; and hydrometallurgical processes for EoL lowquality magnets. Additionally, the Life Cycle Assessment (LCA) and Process Integration with Flowsheet simulation tool will integrate the whole recycling value chain (collection, dismantling, physical and chemical treatment options, and re-manufacturing) and assess the environmental impact of the different processes.

Funding: This activity has received funding from the European Institute of Innovation and Technology (EIT), under project number 19049, in the Upscaling activity, in the thematic field of Recycling and material chain optimization for End-of-Life products at EIT Raw Materials. This body of the European Union receives support from the Horizon 2020, the EU Framework Programme for Research and Innovation.

Conflicts of Interest: The authors declare no conflict of interest.

\section{References}

1. EC. Study on the EU's List of Critical Raw Materials; EC: Brussels, Belgium, 2020.

2. Goonan, T.G. Rare Earth Elements: End Use and Recyclability; US Department of the Interior, US Geological Survey Reston: Reston, VA, USA, 2011.

3. Alonso, E.; Wallington, T.; Sherman, A.; Everson, M.; Field, F.; Roth, R.; Kirchain, R. An assessment of the rare earth element content of conventional and electric vehicles. SAE Int. J. Mater. Manuf. 2012, 5, 473-477. [CrossRef]

4. Hatch, G.P. Dynamics in the global market for rare earth. Elements 2012, 8, 341-346. [CrossRef]

5. Binnemans, K.; Jones, P.T. Is REE recycling the solution for the balance problem? In Proceedings of the DEMETER Closing Symposium "Rare-Earth Permanent Magnet Motors and the e-Mobility Revolution", Leuven, Belgium, 5-7 February 2019.

6. Yang, Y.; Walton, A.; Sheridan, R.; Güth, K.; Gauß, R.; Gutfleisch, O.; Buchert, M.; Steenari, B.-M.; Van Gerven, T.; Jones, P.T. REE recovery from end-of-life NdFeB permanent magnet scrap: A critical review. J. Sustain. Metall. 2017, 3, 122-149. [CrossRef]

7. Yang, Y.; Abrahami, S.; Xiao, Y. Recovery of rare earth elements from EOL permanent magnets with slag extraction. In Proceedings of the 3rd International Slag Valorisation Symposium, Leuven, Belgium, 19-20 March 2013; pp. $249-252$.

8. Diehl, O.; Schönfeldt, M.; Brouwer, E.; Dirks, A.; Rachut, K.; Gassmann, J.; Güth, K.; Buckow, A.; Gauß, R.; Stauber, R. Towards an alloy recycling of Nd-Fe-B permanent magnets in a circular economy. J. Sustain. Metall. 2018, 4, 163-175. [CrossRef]

9. Menad, N.; Seron, A.; Save, M.; Menard, Y.; Maat, N.; Le Breton, J.-M.; Nachbaur, V.; Bizouard, A.; Delain, M.; Waignein, L. Recovery of permanent magnets type NdFeB from WEEE. In Proceedings of the 6th International Conference on Engineering for Waste and Biomass Valorisation, Albi, France, 23-26 May 2016.

10. Walton, A.; Yi, H.; Rowson, N.A.; Speight, J.D.; Mann, V.S.J.; Sheridan, R.S.; Bradshaw, A.; Harris, I.R.; Williams, A.J. The use of hydrogen to separate and recycle neodymium-iron-boron-type magnets from electronic waste. J. Clean. Prod. 2015, 104, 236-241. [CrossRef]

11. Sprecher, B.; Xiao, Y.; Walton, A.; Speight, J.; Harris, R.; Kleijn, R.; Visser, G.; Kramer, G.J. Life cycle inventory of the production of rare earths and the subsequent production of NdFeB rare earth permanent magnets. Environ. Sci. Technol. 2014, 48, 3951-3958. [CrossRef] [PubMed] 